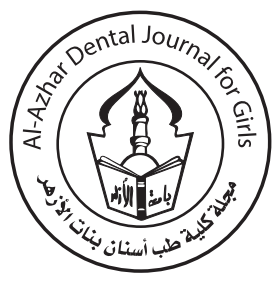

\title{
Immunohistochemical Expression of Cortactin, E-Cadherin, and $M D M 2$ Proteins in Solid Ameloblastoma versus Odontogenic Keratocyst (An immunohistochemical study)
}

\author{
Hanaa M. Abd Elsamia ${ }^{1 *}$, Nadia A. Radi ${ }^{2}$, Eman A. Abo Hager ${ }^{3}$, Adel A. Shouman ${ }^{4}$
}

Codex : 39/1907

azhardentj@azhar.edu.eg

http://adjg.journals.ekb.eg

DOI: $10.21608 /$ adjg.2019.7780.1100

\section{KEYWORDS}

Cortactin, E-Cadherin,

MDM2, Solid Ameloblastoma,

Odontogenic Keratocyst

\begin{abstract}
Purpose: The extent of invasion can be analyzed by the expression and production of various genes and proteins by lesional cells. However, current clinical parameters lack the potential to predict the neoplastic behavior in solid ameloblastoma (SAB) and odontogenic keratocyst (OKC). Cortactin, an F-actin binding protein, overexpression has been correlated with advanced clinic pathological stage and poor prognosis in several tumors. E-cadherin belongs to the classical cadherins which. Low E-cadherin expression correlated to aggressive, poorly differentiated, high-grade carcinomas and low patient survival. Human-murine double minute 2 (MDM2), contributes to the promotion of cell growth, survival, invasion, and therapeutic resistance, overexpression of MDM2 has been observed in various human cancers and can contribute to genomic instability, thus, further promoting tumorigenesis. The purpose of this work was to investigate the role of Cortactin, E-Cadherin, MDM2 proteins expression in SAB and $\mathrm{OKC}$ and correlate the expression of these markers with the aggressive behavior of these tumors. Material and method: 10 case of solid ameloblastoma with its different histologic variants and 10 cases of the keratocystic odontogenic tumor were collected as paraffin embedded blocks. An immunohistochemical investigation using, Cortactin, E-Cadherin, and MDM2 antibodieswere done for all specimens. Results: The mean area percent of immunoexpression of Cortactin was greatest in SAB while, the mean area percent of immunoexpression of E-Cadherin, MDM2 were greatest in OKC. Conculsion: According to the current study, the absence of any significant differences between $\mathrm{AB}$ and $\mathrm{OKC}$ indicate the neoplastic and aggressive nature of $\mathrm{OKC}$ similar to $\mathrm{AB}$.
\end{abstract}

* Paper extracted from $\mathrm{PhD}$ thesis titled "Immunohistochemical Analysis of Cortactin, E-Cadherin, and MDM2 Proteins Expression in Selected Benign and Malignant Ectodermal Odontogenic Tumors.

1. Assistant lecturer of Oral and Dental Pathology. Faculty of Dental Medicine for Girls, Al Azhar University, Cairo, Egypt. email:dr.hanoa@gmail.com

2. Lecturer of Oral and Dental Pathology. Faculty of Dental Medicine for Girls, Al Azhar University, Cairo, Egypt.

3. Professor of Oral and Dental Pathology. Faculty of Dental Medicine for Girls, Al Azhar University, Cairo, Egypt.

4. Professor of Oral and Dental Pathology. Faculty of Dental Medicine for Girls, Al Azhar University, Cairo, Egypt. 


\section{INTRODUCTION}

Odontogenic tumors consist of a diverse group of lesions because tooth development is a complex procedure in which cells, in different stages of differentiation, contribute to the many phenotypes of histological variety in the odontogenic lesions (1). The pathogenesis of odontogenic tumors is not clearly distinct and various cell types may take parts in this process. A multitude of odontogenic tumors originates via some deviation from the normal pattern of odontogenesis which reflects their compound multiformity. It is well-known that harmonized collaborations between the epithelial and stromal cells are important for government the growth and clinical manners of pathoses ${ }^{(2-4)}$. Ameloblastoma (AB) is a benign epithelial odontogenic neoplasm of the jaw with an insidious growth pattern. It is frequently aggressive and destructive, able to achieve great size, corrode bone and invade adjacent tissues ${ }^{(5,6)}$. Odontogenic keratocyst (OKC) is benign uni or multicystic, intrabony cyst of odontogenic source, with unique distinguishing lining of the parakeratinized stratified squamous epithelium. Due to its histopathological variant characteristics, specific clinical course, high recurrence rate, high rate of proliferative activity, invasive behavior and relation to the nevoid basal cell carcinoma syndrome all these features reflect the neoplastic nature of these lesions. The invasion extent can be investigated by the expression of different genes proteins by tumoral cells. However, current clinical parameters lack the potential to anticipate the neoplastic behavior in both $\mathrm{SAB}$ and $\mathrm{OKC}{ }^{(7-9)}$.

Cortactin is an F-actin binding protein (filament) originally known as a substrate for Src family kinases (SFK) ${ }^{(10)}$. Originally, little was known about its function, except that it bounds to actin filaments leading to enhancement of cell motility and loss of cell-cell adhesion this has been accomplished through its involvement in all steps of the invadosome lifecycle, from assembly, maturation, proteolytic activity, and disassembly. Cortactin overexpression was found in different cancers such as head and neck squamous carcinoma, breast, hepatocellular carcinoma, and bladder cancer, and linked with reduced patientprognosis and decline survival. Its overexpression has been corresponding to advanced clinic stage and poor out come in several tumors ${ }^{(11-13)}$

The cadherin family consist of more than 100 members and divided into three subfamilies: classical cadherins, nonclassical cadherins, and protocadherins. Of these, classical cadherins are the greatest widely studied and E-cadherin belongs to the classical cadherins mediating intercellular adhesion at the level of epithelia so maintains the structural and functional integrity of epithelial tissues ${ }^{(14)}$. E-cadherin expression is inversely correlated to the grade of differentiation in different cancers ${ }^{(15,16)}$. On the other hand, E-cadherin is related to aggressive cancer in many organs as, breast cancer, ovarian carcinomas, and glioblastomas, where E-cadherin is linked to decline survival and correlates with enhanced invasiveness ${ }^{(17-20)}$.

The murine double minute 2 (MDM2) is an oncoprotein which share in the promotion of cell proliferation, survival, invasion, and treatment resistance. MDM2 is a negative regulator of the p53 tumor suppressor protein through binding to the transcriptional initiation domain of p53 leading to proteasome-mediated degradation. High expression of MDM2 lead to genomic instability, thus, promoting tumorigenesis ${ }^{(21)}$. Increase expression of MDM2 has been detected in many human malignanciesinvolvingmelanoma, breastcarcinoma, glioblastoma, leukemia ${ }^{(22)}$. Overexpression of MDM2 in ameloblastomas indicates that an increased production or decreased breakdown of protein leads to disturbance in growth regulation (23). To date, no appropriate immunohistochemical marker is presented to estimate the aggressiveness of both $\mathrm{SAB}$ and $\mathrm{OKC}$, therefore, the objective of this study is to assess the association of both Cortactin, E-Cadherin, and MDM2 expression in both $\mathrm{SAB}$ and $\mathrm{OKC}$ and analyzing their correlation with the biological behavior of these neoplasms by means of the immunohistochemical technique. 


\section{MATERIALS AND METHODS}

\section{Case selection}

The specimens for this study were retrieved as paraffin-embedded blocks from the archives of Oral Pathology Department, Faculty of Dentistry, Alexandria University. The specimens were divided into two groups according to WHO classification (10 cases of SAB with its different histologic variants and 10 cases of the OKC.

\section{Histological analysis}

Using H\&E for reevaluation of the abovementioned cases was carried out to confirm their diagnosis, and reclassifying them according to $\mathrm{WHO}^{(24)}$.

\section{Immunohistochemical analysis}

Sections of $4 \mu \mathrm{m}$ thickness were mounted on electrically charged slides and deparaffinized. To block endogenous peroxidase activity, the samples were subjected to $3 \%$ hydrogen peroxide solution for 5 minutes. They were subjected to heat-prompted antigen retrieval procedure using citrate buffer (10mM, pH 6.0) in a pressure cooker for $2 \mathrm{~min}$. Consequently, the tissue sections were incubated with primary monoclonal antibodies according to the manufacturer's directions using Cortactin, E-Cadherin, and MDM2 mouse monoclonal antibodies (Clone A-4 Cat.No.SC-55578; Clone $\mathrm{NCH}-38$, and Clone 1B10). Followed by $45 \mathrm{~min}$ in secondary antibody (rabbit antibody against mouse immunoglobulins) (DAKO, Denmark). It was then immersed in streptavidin-biotin peroxidase conjugate (DAKO) and incubated for $10 \mathrm{~min}$ with 3, 3-diaminobenzidine chromogen (DAKO). Mayer's hematoxylin was used as counter-stain. All the steps were carried out at room temperature and after each step the sections were rinsed with PBS, $\mathrm{pH} 7.2^{(25)}$.

\section{Histomorphometric analysis}

Immunoreactivity, for Cortactin, E-Cadherin, and MDM2; were evaluated by assessing the area percent of positive immunostained cells in relation to the area inspected in each field using image analyzer computer system (Leica, Germany) at Oral and Dental Pathology Department, Faculty of Dental Medicine for Girls, Al Azhar University. Using the color detection, areas of positive immunostaining were covered by a blue binary color. Ten fields per each tissue section of each patient were sequentially taken, to be histomorphometrically assessed. Mean values were then gotten for each tissue section.

\section{Statistical analysis:}

Data were represented as means and standard deviation (SD) values, ANOVA test used to compare means of more than two groups. Tukey-Kramer multiple comparisons were used in the procedure of pairwise comparisons between the groups when the ANOVA test is significant. The $\mathrm{P}$ value is significant if less than or equal to 0.05 ( $\mathrm{P} \leq 0.05)$. Statistical analysis was achieved by means of instate graph pad version 3.10 and Microsoft ${ }^{\circledR}$ excel 2007.

\section{RESULTS}

\section{Histopathological findings:}

SAB has five different histopathological variants. They are follicular ameloblastoma showed multiple nests of odontogenic epithelium. The outer cells are tall columnar with reverse polarity resembling ameloblasts and central stellate reticulum like cells surrounded by connective tissue stroma. These islands sometimes surrounded by juxta follicular hyalinized zone of fibrous connective tissue. Cystic degeneration in the central stellate reticulum- like cells of the follicles resulted in the formation of microcysts was observed in these islands (Fig. 1; A), while in acanthomatous variant the neoplastic island 
showed central stellate reticulum like cells with formation of squamous cells with eosinophilic cytoplasm (Fig. 1; B), basal cell ameloblastoma composed of islands of uniform compacted dark stained cuboidal basaloid cells surrounded by juxtaepithelial hyalinized homogenous fibrous tissue band(Fig. C), granular cell ameloblastoma, showed tumor islands with peripheral columnar cells surrounding central large cells with granular eosinophilic cytoplasm and peripheral nuclei (Fig. 2;M) and plexiform ameloblastoma showed anastomosing cords of ameloblastoma epithelium, the peripheral cells are cuboidal and the central cells are few loosely arranged stellate reticulum like cells within degenerated connective tissue stroma (Fig. $2 ; \mathrm{N}$ ).

OKC showed cystic lumen lined by thin uniform epithelium without rete pegs. It surrounded by connective tissue wall containing daughter cyst. There is an area of detachment of a portion of epithelial lining from the fibrous connective tissue wall (Fig. 2; O).

\section{Immunohistochemical findings:}

Cortactin immunostaining was seen in the cytoplasm and/or cell membrane of the outer basal and stellate-reticulum like cells of the neoplastic islands of all histopathological variants of solid ameloblastoma while it was negative in central squamous cells of acanthomatous type (Fig. 1; D, E, F) (Fig. 2; P, Q). While in OKC cortactin immunostaining was seen in the cytoplasm and/ or cell membrane of the entire epithelial lining.
Few connective tissue fibroblasts showed positive immunostaining (Fig. 2; R).

E-cadherin immunostaining was seen on the cell membrane and less frequently cytoplasmic chiefly of central stellate- reticulum like cells. Few outer cells of the neoplastic nests may show positive immunostaining in all histopathological variants of solid ameloblastoma except basal cell ameloblastoma, immunostaining was membranes of the basaloid cells of the neoplastic island (Fig. 1; G, H, I) (Fig.2; S, T). While in OKC E-cadherin immunostaining was seen on the cell membrane of suprabasal layers, while it was negative in the basal cell layer and parakeratinized surface (Fig. 2; U).

MDM2 immunostaining was seen in the nucleus and cytoplasm of cells of the neoplastic islands including all histopathological variants of solid ameloblastoma while it was negative in the central squamous cells of acanthomatous type (Fig. 1; J, K, L) (Fig. 2; V, W). While in OKC, MDM2 immunostaining was seen on the nucleus and cytoplasm of all layers of epithelial lining. Some connective tissue fibroblasts showed positive nuclear immunostaining (Fig. 2; X).

\section{Statistical Results}

\section{Comparison between SAM and OKC}

The statistical difference between solid ameloblastoma and odontogenic Keratocyst was not significant considering the three markers as showed in (Table. 1,2, 3\& Fig. 3,4,5). 


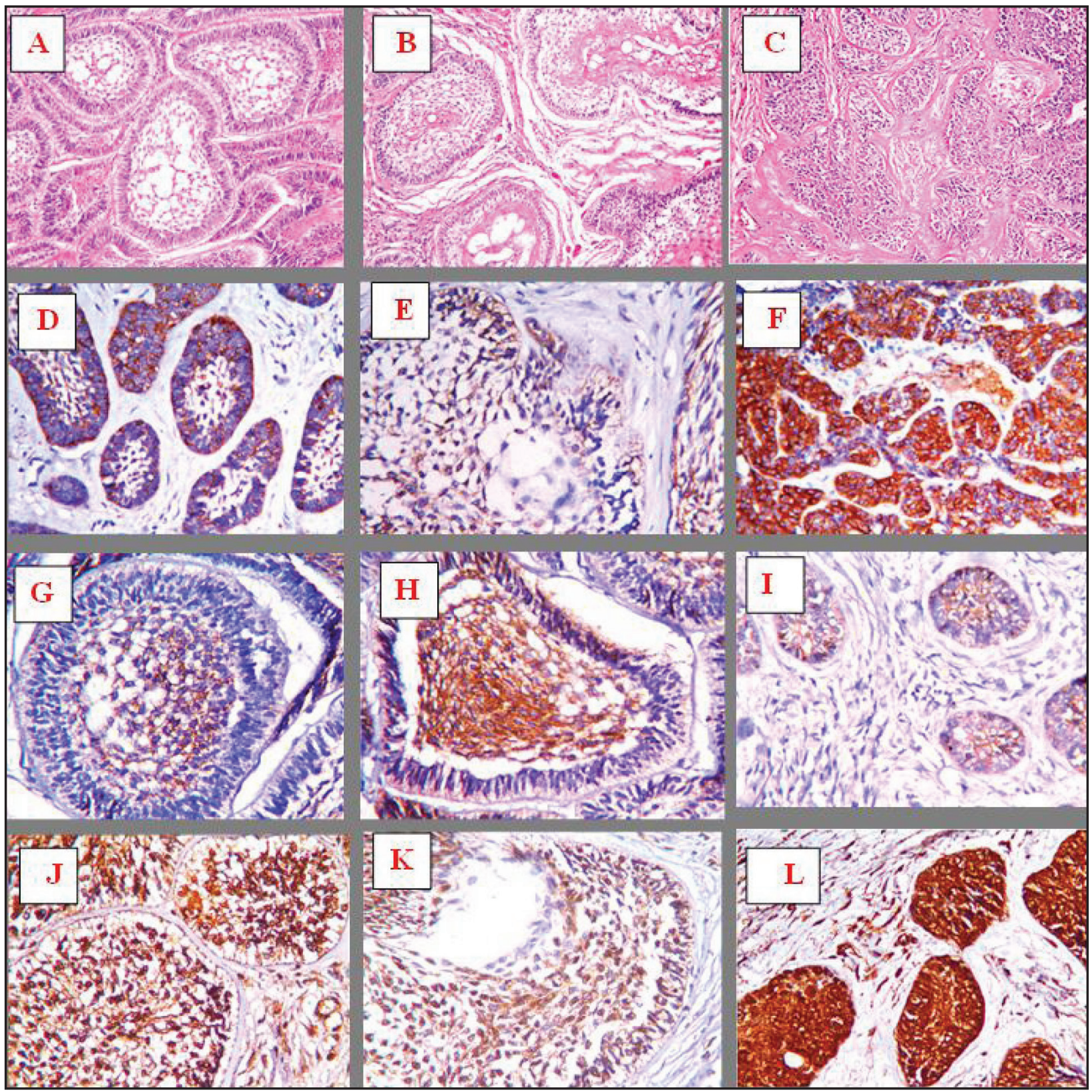

Figure(1) Follicular ameloblastoma showed multiple islands of odontogenic epithelial cells A, acanthomatous variant, the neoplastic island showed central stellate reticulum like cells with formation of squamous cells $\mathbf{B}$, basal cell ameloblastoma composed of islands of uniform compacted dark stained cuboidal basaloid cells $\mathbf{C},(\mathbf{A}-\mathbf{C}, \mathbf{H} \& \mathbf{E} \mathbf{X} 200)$. Cortactin immunostaining was seen on cytoplasm and/or cell membrane of follicular, acanthomatous, and basal cell variants of AB (D-F, cortactin, X200). E-cadherin immunostaining was seen on cell membrane and cytoplasm mainly of central stellate- reticulum like cells of follicular and acanthomatous, while in basal cell ameloblastoma, showed membronus expression of the basaloid cells (G-I, E-cadherin, X 200). MDM2 immunostaining was seen on the nucleus and cytoplasm of peripheral cells and central stellate reticulum like cells of follicular, acanthomatous, and basal cell variants of $\mathrm{AB}$, while it was negative in the central squamous cells of acanthomatous type (J-L, MDM2, X 200). 


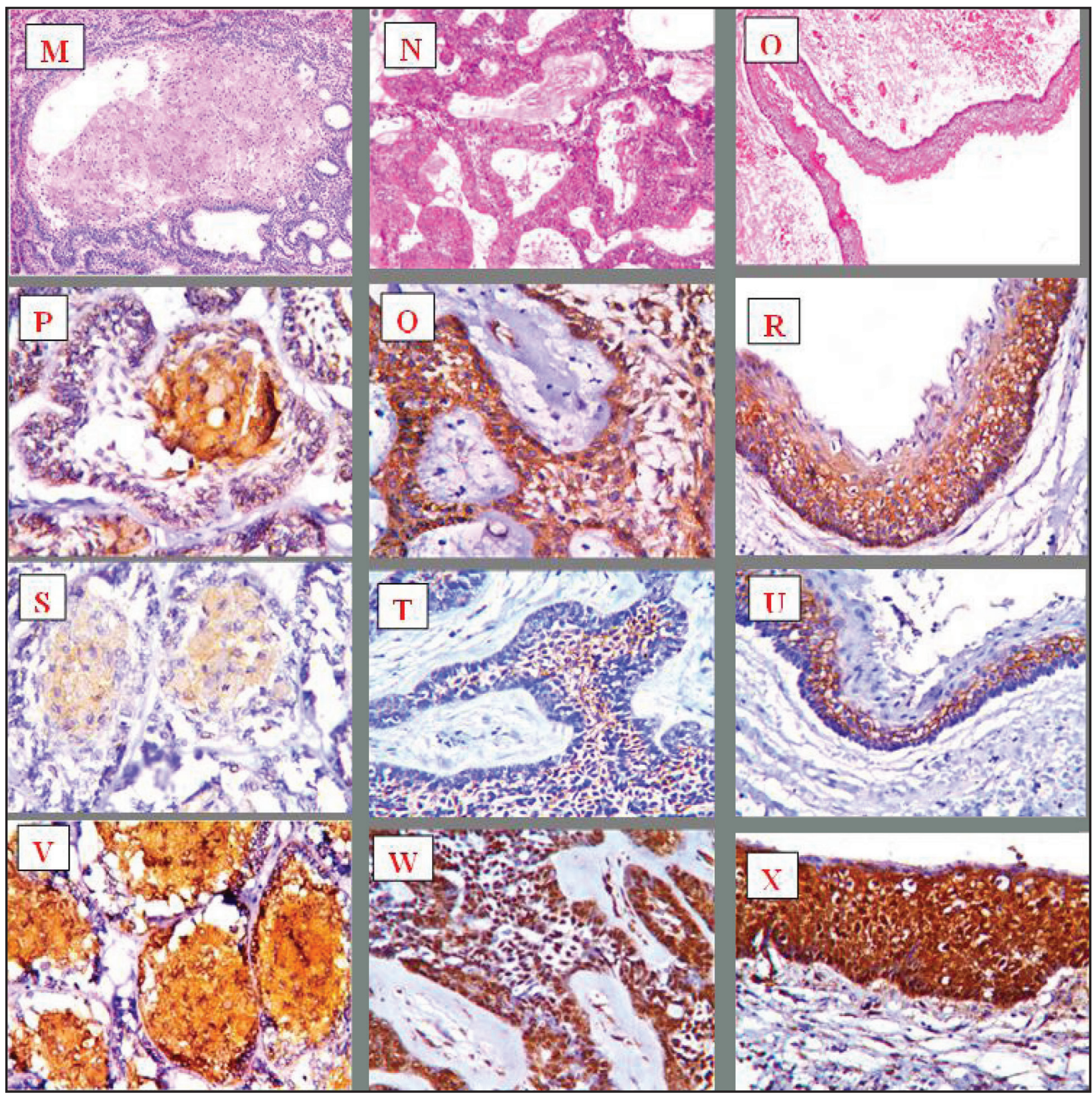

Figure (2) Granular cell ameloblastoma, showed tumor islands with peripheral columnar cells surrounding central large cells with granular eosinophilic cytoplasm and peripheral nuclei $\mathbf{M}$, plexiform ameloblastoma showed anastomosing cords of ameloblastoma epithelium $\mathbf{N}$, OKC, showed cystic lumen lined by thin uniform epithelium without rete pegs $\mathbf{O}$ (N-O, H\&E $\mathbf{X}$ 200), Cortactin immunostaining was seen on cytoplasm and/or cell membrane of granular $\mathbf{P}$, and plexiform $\mathbf{Q}$ variants of $\mathrm{AB}$. OKC cortactin immunostaining was seen on cytoplasm and/or cell membrane of all layers of epithelial linning R. (P-R, cortactin, X200). ). E-cadherin immunostaining was seen on cell membrane and cytoplasm mostly of central stellate- reticulum like cells of granular $\mathbf{S}$, and plexiform $\mathbf{T}$, variants of $\mathrm{AB}$ while in $\mathrm{OKC}$ E-cadherin immunostaining was seen on cell membrane of supra basal layers, but it was negative in the basal cell layer and parakeratinized surface U, (S-U, E-cadherin, X 200). MDM2 immunostaining was seen on the nucleus and cytoplasm of peripheral cells and central stellate reticulum like cells of neoplastic islands of granular $\mathbf{V}$, and plexiform $\mathbf{W}$, variants of AB. While in OKC, MDM2 immunostaining was seen on the nucleus and cytoplasm of all layers of epithelial lining X, (V-X, MDM2, X 200). 
Table (1) Comparison between cortactin area \% in SAM\&OKC

\begin{tabular}{|l|c|c|c|c|c|c|}
\hline \multicolumn{1}{|c|}{ Tumor type } & Mean & SD & Median & Minimum & Maximum & \\
\hline Solid ameloblastoma & $37.47^{\mathrm{B}}$ & 5.80 & 36.01 & 29.91 & 44.13 & $<0.001 *$ \\
\hline Keratocystic odontogenic tumor & $37.22^{\mathrm{B}}$ & 2.90 & 38.41 & 33.21 & 40.42 & \\
\hline
\end{tabular}

*: Significant at $P \leq 0.05$

Table (2) Comparison between E-cadherin area \% in SAM\&OKC

\begin{tabular}{|l|c|c|c|c|c|c|}
\hline Tumor type & Mean & SD & Median & Minimum & Maximum & \\
\hline Solid ameloblastoma & $14.14^{\mathrm{B}}$ & 1.93 & 13.99 & 11.72 & 16.56 & $<0.001^{*}$ \\
\hline Keratocystic odontogenic tumor & $14.93^{\mathrm{B}}$ & 2.93 & 14.58 & 11.30 & 19.78 & \\
\hline
\end{tabular}

*: Significant at $P \leq 0.05$

Table (3) Comparison between MDM2 area \% in SAM\&OKC

\begin{tabular}{|l|c|c|c|c|c|c|}
\hline Tumor type & Mean & SD & Median & Minimum & Maximum & \\
\hline Solid ameloblastoma & $39.91^{\mathrm{B}}$ & 1.90 & 39.81 & 37.59 & 42.10 & $<0.001 *$ \\
\hline Keratocystic odontogenic tumor & $40.94^{\mathrm{B}}$ & 3.63 & 40.97 & 35.81 & 45.76 & \\
\hline
\end{tabular}

*: Significant at $P \leq 0.05$

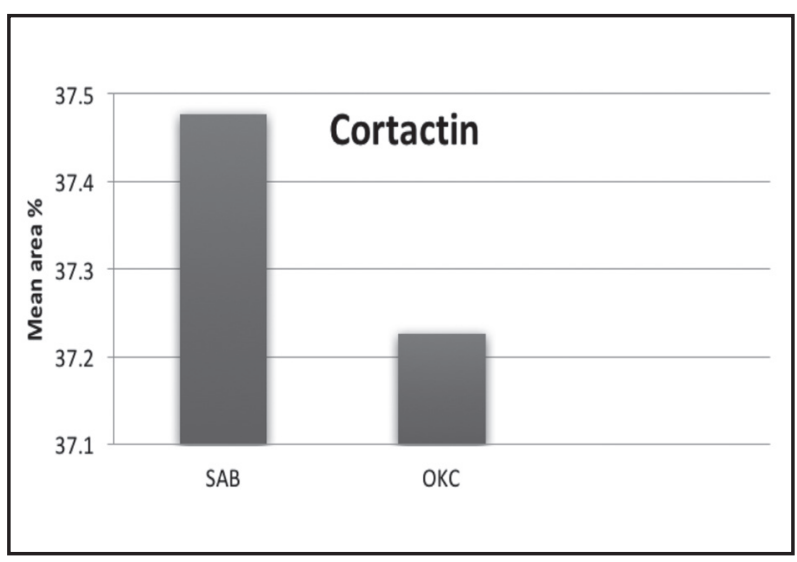

Figure (3) Bar chart representing mean values for cortactin area $\%$ in $\mathrm{SAB}$ and $\mathrm{OKC}$.

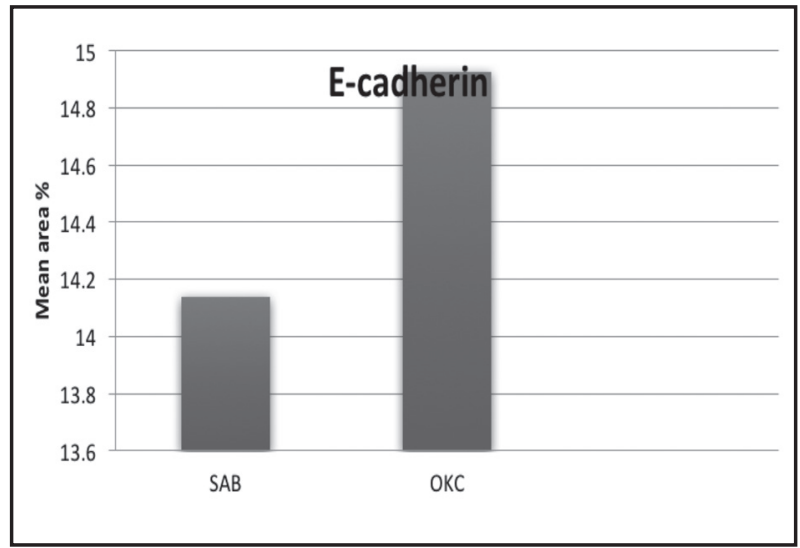

Figure (4) Bar chart representing mean values for E-cadherin area $\%$ in $\mathrm{SAB}$ and $\mathrm{OKC}$. 


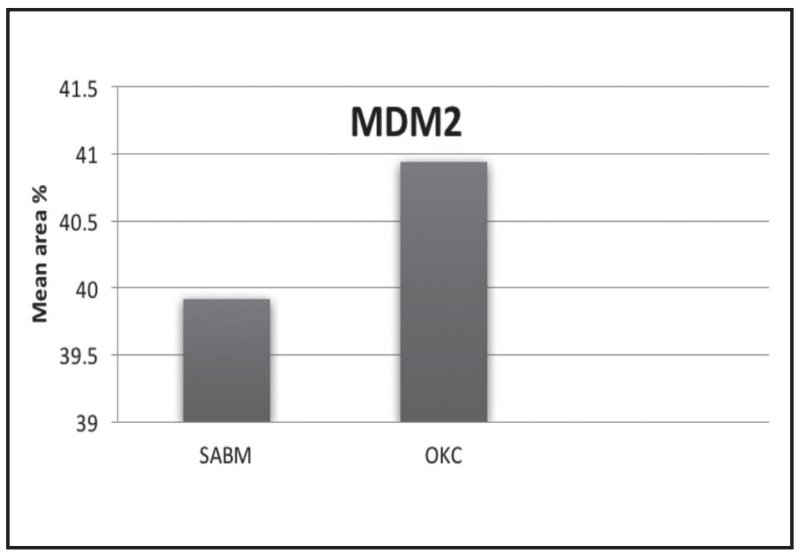

Figure (5) Bar chart representing mean values for MDM2 area $\%$ in $\mathrm{SAB}$ and $\mathrm{OKC}$

\section{DISCUSSION}

Since the prognosis of various neoplasms including odontogenic tumors cannot be reliably and precisely predicted on the basis of clinical and histopathologic features only, it is highly desirable to find genetic markers to rely on. The last could be an objective measure to gain insight into alterations that might be occurring during the process of tumorigenesis. So, the existing study was designed to assess the immunohistochemical expression of cortactin, E-cadherin and MDM2 in SAB and $\mathrm{OKC}$, correlate their expression to each other and to predict their prognostic value in these tumors.

Regarding SAB, cortactin immunostaining was detected in the peripheral and central stellate cells of the tumoral epithelial nests while it was negative for squamous area and keratin in the acanthomatous subtype. These results were in accordance with other studies that showed acanthomatous epithelial tumors were non-reactive for invadopodia proteins and they explained this as tumor areas with strong invadopodia protein expression would related to sites of dynamic actin remodeling while nonexpression mean a shift to dormancy ${ }^{(26,27)}$. Also, cortactin immunolocalization was cytoplasmic and membranous. This finding was explained as cortactin directed to the cell membrane to induce invadopodia assembly. In addition, cortactin could attach to actin-related molecules complexes that might act as bridges in the early steps of invadopodia development. It was revealed that local invasiveness of ameloblastoma is rely on the migrant potential of tumor cells as defined by their allocation of cortactin and other invadopodia proteins. It was suggested that cell migration and extracellular matrix destruction affect ameloblastoma behavior and that these cellular activities are concomitant with cortactinintermediated invadopodia development and membrane-type 1 MMP (MT-MMP) enrolment ${ }^{(28-30)}$.

Regarding OKC, cortactin immunostaining was seen as cytoplasmic and membranous localization in all layers of OKC. These results were in accordance with other studies ${ }^{(31,32)}$. It was reported that $\mathrm{OKC}$ is a multicystic neoplasm, which could show daughter cysts that occur sporadically as invadopodia may share in this process due to its capability to facilitate local invasion. Invadopodia pericellular activity possibly lead to separation of one or more tumor cells from the primary cyst, result in creation of daughter cyst and this occurrence supported by increase expression of invadopodia-associated proteins like cortactin and MT1-MMP in OKC ${ }^{(28)}$.

In the present study, the distributions of positive immune reaction were variable among the studied tumors, where there no statistically significant difference between SAB and OKC. This finding was in agreement with another result that revealed expression of podoplanin which modulate the actin cytoskeleton and act as a mediator in the pathway of cellular invasion was expressed in OKC and ameloblastomas with no significant difference ${ }^{(33)}$. Invadopodia proteins have been recognized as a principle element of the crosstalk between tumor cells and their surrounding microenvironment. Significant to their role in promoting tumor progress, invasion and metastases are their capacity to orchestrate actin cytoskeleton remodeling in tumoral as well as stromal parts. Tumor cells can affect the contractile property of stromal cells. Enlarged stromal cell contractility result in high matrix rigidity and this has tumor- enhancing effects. It is likely that the noticed various distribution 
form for cortactin in ameloblastoma epithelial and stromal cells could reflect the various steps of actin turnover.It was found that although $\mathrm{SAB}$ and $\mathrm{OKC}$ exhibit slow growth; however, they have local invasive manners and high rate of recurrence if not treated with more aggressive actions ${ }^{(27,34-36)}$.

Regarding $\mathrm{SAB}$, in the current study, the distributions of immune reaction for E-cadherin were noted on the cell membrane mainly in the central stellate reticulum-like cells than peripheral columnar cells of ameloblastoma. These results are agreed with previous results which demonstrated that the pattern of E-cadherin immunoreactivity was predominantly on the membrane at cell-cell borders and the most intense reactivity was noticed in the stellate-reticulum like cells. The intensity decreased in the outer columnar cells, especially at the invasive head. This indicates that the outer cells of ameloblastoma display EMT and have the

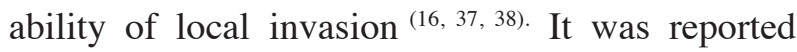
that the immunoreaction for E-cadherin in central parts of tumor nests in SAB that morphologically like to the stellate reticulum of the enamel organ propose that this neoplastic epithelial element preserve the characteristics of cytodifferentiation of the odontogenic epithelium ${ }^{(39)}$. Other study revealed the highest E-cadherin expression in the stellate reticulum like cells, suggested a possible higher level of this protein at certain areas where it enhance the adhesion between the distant cells in this organization regarded as a compensatory phenomenon that is an attempt to maintain normal cellular architecture ${ }^{(40)}$.

On the other side, it was found that E-cadherin reaction was weak or disappear in the squamous metaplastic areas and keratinized areas of acanthomatous ameloblastomas, due to end differentiation of the tumor cells. Also, in granular ameloblastoma, E-cadherin expression was weak cytoplasmic and membranous in clusters of granular cells as these cells have high apoptotic reaction in comparison with other types of ameloblastoma. These results might be due to end differentiation of the tumor cells such as degenerative and/ or maturation changes and were not consider progression or malignant potential of the tumors ${ }^{(37) \text {. }}$

Concerning $\mathrm{OKC}$, in the current study, the distributions of positive immune reaction for E-cadherin were observed on the cell membrane mainly of suprabasal layers. These results were in accordance with another study ${ }^{(39)}$ described the higher reaction of E-cadherin in the suprabasal layers of the epithelial lining of $\mathrm{OKC}$, representing a high degree of cell adhesion between epithelial cells of these layers of the tumor. While, a moderate to high-grade loss of E-cadherin staining in all tissue samples of OKC was found and explained as a down-regulation of $\beta$-catenin and E-cadherin in basal and luminal parakeratinized cell layers of $\mathrm{OKC}$, indicating an invasive potential ${ }^{(41)}$.

In the current study, the mean value of area percent of E-cadherin immunostaining was higher in OKC than SAB with no statistically significant difference between them. These results are in line with other's results ${ }^{(42)}$ confirmed that adhesion molecules as (CD166) were expressed in ameloblastoma and OKC with no statistically significant difference between them. This could be explained by decreased to the negative reaction of E-cadherin in peripheral basal cells of both lesions where they express EMT phenotype and have the potential of focal invasion. The E-cadherin complex redistributation from the cell membrane to cytoplasm explained by failure of E-cadherin and the catenins to localize to the cell membrane and/or bind the cytoskeleton regardless their plenty may be due to genetic or epigenetic alterations in their structure and/or function. Another explanation for cytoplasmic expression pattern instead of cell membrane reaction of E-cadherin is abnormal tumor-related alteration rather than loss or reduction of expression ${ }^{(43)}$.

Regarding $\mathrm{SAB}$, the positivity for MDM2 was observed in nucleus and cytoplasm of the outer and central stellate reticulum like cells. These results were in agreement with a previous study (23) representing the expression of MDM2 was almost confined to nuclei except on occasions 
cytoplasmic staining was observed. The MDM2 positive nuclei were found predominantly in peripheral ameloblast-like cells and it was also seen in central stellate reticulum- like cells suggesting that the peripheral cells have an anti-apoptotic and proliferative phenotype than the central cells. This also, in accordance with other study stated that the proliferative activity of ameloblastomas is nearly limited to the outer layer of the epithelial islands ${ }^{(44)}$.

Regarding OKC, it showed positive cytoplasmic and nuclear immunostaining of MDM2 in all layers of epithelial lining suggesting a neoplastic origin for $\mathrm{OKC}$ and distinct biological behavior than the other non-neoplastic odontogenic cysts, as for proliferation, apoptosis and differentiation processes $^{(45)}$.

In the present study, the mean area percent of MDM2 immunostaining was higher in OKC than $\mathrm{SAB}$ without significant difference between them. These results agreed with previous study found that the reaction of MDM2 was high in ameloblastoma and $\mathrm{OKC}$ without significant difference ${ }^{(45)}$. It was suggested that increased reaction of MDM2 proteins plays a role in pathogenesis and tumor development of SAB and $\mathrm{OKC}$, and this amplified expression give an explanatory mechanism for the likenesses in the biologic behavior of the two lesions and their activity in the same way.Moreover, these results go with other outcomes suggested that $\mathrm{OKC}$ and $\mathrm{SAB}$ have similar proliferation indices in the basal compartment whereas, in the suprabasal compartment, OKC has a higher proliferative capacity. This calls for an aggressive and more radical treatment for $\mathrm{OKC}$ too than just a simple enucleation as is usually performed ${ }^{(46)}$.

\section{CONCLUSION}

From the previous results, we can conclude that Cortactin can be used as a potential invasive marker to differentiate between aggressive and nonaggressive odontogenic tumors as well as the absence of any significant differences between $\mathrm{SAB}$ and $\mathrm{OKC}$ indicate the aggressive nature of $\mathrm{OKC}$.

\section{REFERENCES}

1. Khalele BAEO. The anecdote of viral etiopathogenic in ameloblastoma and odontogenic keratocyst: Why don't we let it go? J Oral Biol Craniofac Res. 2017; 7: 101-5.

2. Regezi JA, Sciubba JJ, Jordan RC. Oral Pathology: Clinical Pathologic Correlations. 5th ed. St. Louis: Saunders Elsevier; 2011. 261-71.

3. Ravikanth M, Soujanya P, Manjunath K, Saraswathi TR, Ramachandran CR. Heterogeneity of fibroblasts. J Oral Maxillofac Pathol. 2011; 15: 247-50.

4. KouhsoltaniM,HalimiM,JabbariG.Immunohistochemical evaluation of myofibroblast density in odontogenic cysts and tumors. J Dent Res Dent Clin Dent Prospects. 2016; 10: $37-42$.

5. Milman T, Ying GS, Pan W, LiVolsi V. Ameloblastoma: 25 Year experience at a single institution. Head Neck Pathol. 2016; 10: 513-20.

6. Ghattamaneni S, Nallamala S, Guttikonda VR. Unicystic ameloblastoma in conjunction with peripheral ameloblastoma: A unique case report presenting with diverse histological patterns. J Oral Maxillofac Pathol. 2017; 21: 267-72.

7. Kebede B, Dejene D, Teka A, Girma B, Aguirre EP, Guerra NEP. Big Keratocystic Odontogenic Tumor of the Mandible: A Case Report. Ethiop J Health Sci. 2016; 26: 491-6.

8. Karthikeyan Maruthamuthu, G. Vasupradha, Janardhanam Dineshshankar, Abishek Rajaram Balaji. Recurrent keratocystic odontogenic tumor of right maxillary sinus involving the right infraorbital rim. Natl J Maxillofac Surg. 2017; 8: 70-4.

9. Sadri D, Shahsavari F, Farhadi S, Shahabi Z, Mehran Z. Angiogenesis concept in odontogenic keratocyst: A comparative study. Indian J Dent Res. 2017; 28: 275-80.

10. Liang X, Budnar S, Gupta S, Verma S, Han SP, Hill MM et al. Tyrosine dephosphorylated cortactin downregulates contractility at the epithelial zonula adherens through SRGAP1. Nat Commun. 2017; 8: 790-9.

11. Martini V, Gattazzo C, Frezzato F, Trimarco V, Pizzi M, Chiodin G, et al. Cortactin, a Lyn substrate, is a checkpoint molecule at the intersection of BCR and CXCR4 signaling pathway in chronic lymphocytic leukaemia cells. Br J Haematol. 2017; 178: 81-93.

12. MacGrath SM, Koleske AJ. Cortactin in cell migration and cancer at a glance. J Cell Sci. 2012; 125: 1621-6. 
13. Radhakrishnan VM, Kojs P, Young G, Ramalingam R, Jagadish B, Mash EA, et al. pTyr421 Cortactin is overexpressed in colon cancer and is dephosphorylated by curcumin: Involvement of non-receptor type 1 protein tyrosine phosphatase (PTPN1). PLoS One. 2014; 22: 57-96.

14. Liu X, Chu KM. E-cadherin and gastric cancer: cause, consequence, and applications. Biomed Res Int. 2014; 14: $63-8$.

15. Özcan A, Yavan İ, Günhan Ö. Immunohistochemical characteristics of cystic odontogenic lesions: a comparative study. Turk Patoloji Derg. 2015; 31: 104-10.

16. Mello LA, Figueiredo AL, Ramos EA, Gurgel CA, Martins MD, de Figueiredo CR, et al. CD1a-positive Langerhans cells and their relationship with E-cadherin in ameloblastomas and keratocystic odontogenic tumors. J Oral Pathol Med. 2013; 42: 454-61.

17. Kleer CG, van Golen KL, Braun T, Merajver SD. Persistent E-cadherin expression in inflammatory breast cancer. Modern Pathology. 2001;14: 458-64.

18. Lewis-Tuffin LJ, Rodriguez F, Giannini C, Scheithauer B, Necela BM, Sarkaria JN. Misregulated E-cadherin expression associated with an aggressive brain tumor phenotype. PLoS One. 2010; 5: 136-65.

19. Rodriguez FJ, Lewis-Tuffin LJ, Anastasiadis PZ. E-cadherin's dark side: Possible role in tumor progression. Biochimica et Biophysica Acta. 2012;18: 23-31.

20. Eliah R. Shamir, Andrew J. Ewald. Adhesion in mammary development: novel roles for E-cadherin in individual and collective cell migration. Curr Top Dev Biol. 2015; 112: 353-82.

21. Saadatzadeh MR, Elmi AN, Pandya PH, BijangiVishehsaraei K, Ding J, Stamatkin CW, et al. The role of MDM2 in promoting genome stability versus instability. Int J Mol Sci. 2017;18: 181-216.

22. Roszak A, Misztal M, Sowińska A, Jagodziński PP. Murine double minute 2 homolog single nucleotide polymorphisms 285 and 309 in cervical carcinogenesis. Mol Diagn Ther. 2015; 19: 235-44.

23. Krishna A, Kaveri H, Naveen Kumar RK, Kumaraswamy KL, Shylaja S, Murthy S. Overexpression of MDM2 protein in ameloblastomas as compared to the adenomatoid odontogenic tumor. J Cancer Res Ther. 2012; 2: 232-7.

24. EL Naggar AK, Chan JKC, Grandis JR, Takata T, Slootweg PJ, WHO classification of tumors of the head and neck. 4th ed. Lyon: IARC Press; 2017.
25. Ramos-Vara JA, Miller MA. Technical aspects of immunohistochemistry. Vet Pathol. 2014; 51:42-87.

26. Stevenson RP, Veltman D, Machesky LM. Actin-binding proteins in cancer progression at a glance. J Cell Sci. 2012;125: 1073-9.

27. Beaty BT, Condeelis J. Digging a little deeper: the stages of invadopodium formation and maturation. Eur $\mathrm{J}$ Cell Biol. 2014; 93: 438-44.

28. Ribeiro Ribeiro AL, da Costa NM, de Siqueira AS, Brasil da Silva W, da Silva Kataoka MS, Jaeger RG, et al. Keratocystic odontogenic tumor overexpresses invadopodia-related proteins, suggesting invadopodia formation. Oral Surg Oral Med Oral Pathol Oral Radiol. 2016;122: 500-8.

29. Siar CH, Rahman ZA, Tsujigiwa H, Mohamed Om Alblazi K, Nagatsuka H, Ng KH. Invadopodia proteins, cortactin, N-WASP and WIP differentially promote local invasiveness in ameloblastoma. J Oral Pathol Med. 2016; 11: $12-41$.

30. Pinheiro JJV, Nascimento CF, Freitas VM, de Siqueira AS, Junior SM, Jaeger RG. Invadopodia proteins, cortactin and membrane type I matrix metalloproteinase (MT1-MMP) are expressed in ameloblastoma. Histopathology. 2011; 59: 1266-9.

31. Courtneidge SA. Cell migration and invasion in human disease: the Tks adaptor proteins. Biochem Soc Trans. 2012; 40:129-32.

32. Burger KL, Learman BS, Boucherle AK. Src-dependent Tks5 phosphorylation regulates invadopodia-associated invasion in prostate cancer cells. Prostate. 2014; 74:134-48.

33. Singhal N, Khanduri N, Kurup D, Gupta B, Mitra P, Chawla R. Immunohistochemical evaluation of podoplanin in odontogenic tumors and cysts using antihuman podoplanin antibody. J Oral Biol Craniofac Res. 2017; 7: 95-100.

34. Samuel MS, Lopez JI, McGhee EJ. Actomyosin-mediated cellular tension drives increased tissue stiffness and betacatenin activation to induce epidermal hyperplasia and tumor growth. Cancer Cell. 2011; 19: 776-91.

35. Murphy DA, Courtneidge SA. The 'ins' and 'outs' of podosomes and invadopodia: characteristics, formation, and function. Nat Rev Mol Cell Biol. 2012; 12: 413-26.

36. Gadipelly S, Reddy VB, Sudheer M, Kumar NV, Harsha G. Bilateral calcifying odontogenic cyst: A Rare Entity. J Maxillofac Oral Surg. 2015;14: 826-31. 
37. Florescu A, Mărgăritescu C, Simionescu CE, Stepan A. Immunohistochemical expression of MMP-9, TIMP-2, E-cadherin and vimentin in ameloblastomas and their implication in the local aggressive behavior of these tumors. Rom J Morphol Embryol. 2012; 53: 975-84.

38. Fengyu Hao, Jie Liu, Ming Zhong, Junting Wang, Jingdong Liu. Expression of E-cadherin, vimentin, and $\beta$-catenin in ameloblastoma and association with clinicopathological characteristics of ameloblastoma. Int J Clin Exp Pathol. 2018;11: 199-207.

39. Kusafuka K, Hirobe K, Wato M, Tanaka A, Nakajima T. CD56 expression is associated with neuroectodermal differentiationinameloblastomas:animmunohistochemical evaluation in comparison with odontogenic cystic lesions. Med Mol Morphol. 2011;44: 79-85.

40. Jang YJ, Kim HG, Koo TW, Chung PS. Localization of ZO-1 and E-cadherin in the nasal polyp epithelium. Eur Arch Otorhinolaryngol. 2002; 259: 465-9.

41. Hakim SG, Kosmehl H, Sieg P, Trenkle T, Jacobsen HC, Attila Benedek G, et al. Altered expression of cell-cell adhesion molecules $\beta$-catenin/E-cadherin and related Wntsignaling pathway in sporadic and syndromal keratocystic odontogenic tumors. Clin Oral Investig. 2011;15: 321-8.
42. Andisheh-Tadbir A, Gorgizadeh A. CD166 expression in the dentigerous cyst, keratocystic odontogenic tumor and ameloblastoma. J Clin Exp. 2016; 8: 236-40.

43. Gao S, Eiberg H, Krogdahl A, Liu CJ, Sørensen JA. Cytoplasmic expression of E-cadherin and beta-Catenin correlated with $\mathrm{LOH}$ and hypermethylation of the APC gene in oral squamous cell carcinomas. J Oral Pathol Med. 2005; 34: 116-9.

44. Ganvir SM, Khobragade PG, Bamane SA, Kumavat R, Dalmia A. Role of podoplanin expression in deciding the invasive potential of ameloblastoma - A retrospective IHC study. J Oral Biol Craniofac Res. 2016; 6: 187-93.

45. Sharifi-Sistani N, Zartab H, Babakoohi S, Saghravanian $\mathrm{N}$, Jamshidi S, Esmaili H, et al. Immunohistochemical comparison of the expression of p53 and MDM2 proteins in ameloblastomas and keratocystic odontogenic tumors. J Craniofac Surg. 2011; 22: 1652-6.

46. Varsha B, Gharat AL, Nagamalini B, Jyothsna M, Mothkur ST, Swaminathan U. Evaluation and comparison of expression of p63 in odontogenic keratocyst, solid ameloblastoma and unicystic ameloblastoma.J Oral Maxillofac Pathol. 2014;18: 223-8. 